\title{
Variation of Salivary IgA During Weight Loss Period Before a Competition Among University Judo Players
}

\author{
Hiroaki Hiraoka ${ }^{\mathrm{a}}$, Yukichi Hanaoka ${ }^{\mathrm{b}}$, Subrina Jesmin ${ }^{\mathrm{a}}$, Fuminori Kimura ${ }^{\mathrm{a}}$, \\ Yujiro Matsuish ${ }^{\mathrm{a}}$, Kazuhiro Shimizuc ${ }^{\mathrm{c}}$, Koichi Watanabe ${ }^{\mathrm{a}, \mathrm{d}}$
}

\begin{abstract}
Background: This study aims to investigate the health condition of university judo athletes during a period of weight loss before a competition, using secretory immunoglobulin $\mathrm{A}$ (SIgA) in saliva.

Methods: The subjects were 30 university judo athletes, who were divided into three groups: control group $(\mathrm{n}=10)$, under $5 \%$ weight loss group $(n=10)$, and over $5 \%$ weight loss group $(n=10)$. The items evaluated were body weight measurements, salivary SIgA level and its secretion rates, incidences of symptoms of upper respiratory tract infections, and mental state.

Results: The over 5\% body weight loss group had significantly lower SIgA secretion rates on the first day and third day before the real competition compared to those of the control group. Furthermore, the over $5 \%$ body weight loss group had an increased number of symptoms of upper respiratory tract infections. Alterations in mental statuses were also seen, such as decreased vitality and increased fatigue on the day before the competition in the over $5 \%$ weight loss group.
\end{abstract}

Conclusions: This study shows that over $5 \%$ body weight loss in judo athletes induces a decrease in the salivary SIgA secretion rate and causes health condition of the body and the mind to deteriorate.

Keywords: Salivary immunoglobulin A; Judo; Weight loss; Competition; Immunity

\section{Introduction}

Judo, an Olympic sport done all over the world, is a physically demanding combat sport, and this sport has a potential stage to reduce body weight to achieve the respective weight

Manuscript submitted September 11, 2019, accepted October 17, 2019

aFaculty of Health and Sport Sciences, University of Tsukuba, Ibaraki, Japan ${ }^{b}$ Waseda Institute for Sport Sciences, Saitama, Japan

cJapan Institute of Sports Sciences, Tokyo, Japan

${ }^{\mathrm{d} C}$ Corresponding Author: Koichi Watanabe, Faculty of Health and Sport Sciences, University of Tsukuba, 1-1-1, Tennodai, Tsukuba, Ibaraki 305-8575, Japan. Email: watanabe.koichi.ga@u.tsukuba.ac.jp

doi: https://doi.org/10.14740/jocmr3998 classes before the competition starts. In all official competitions, judo athletes are paired with opponents of similar body weight through weight classes to ensure fairness of the game. Although a number of judo athletes reduce body weight before the competition, the weight loss phase induces a lot of changes ranging from basal metabolism, muscular glycogen, aerobic capacity, cardiac output, to the immunological system [1]; especially drastic body weight reduction before competition caused impaired physical performance in judo athletes [2]. Since weight reduction rate $>6 \%$ adversely affects the performance and health of the judoka [3], the less than 5\% weight is desirable for judo athletes to maintain and exert highly developed physical fitness and technical/tactical skills [4].

However, judo players reduce body weight several times per season, and the magnitude of weight cycling is about $5 \%$ to $10 \%$ of body weight [5]. If the weight reduction occurs at a rapid rate before a real competition, a lot of stress and harmful consequences take place inside the body [6-8], and these are particularly important to the conditioning at the time of the weight loss [3]. Although much less attention has been given to judo, recent studies have shown that the patterns and styles of rapid weight loss in judo before completion are very similar and comparable to those reported in wrestling [9]. Wrestlers usually reduce their body weight at a considerable level a few days before competitions at aiming to achieve an advantage against lighter, smaller, and weaker opponents [10-13]. Thus, focused attention has been paid to judo athletes in understanding the more in-depth condition and approaches during the weight loss phase.

Very recent studies have shown the significant comptonization of the immunological system during the weight loss phase of judo players. When a pathogen causes upper respiratory tract infection (URTI), there is an evident outpouring of secretory immunoglobulin A ( $\operatorname{SIgA})$, a potential and established marker of protection against URTI [14] by preventing viral replication, inhibiting the attachment of bacteria and viruses at mucosal epithelium in the mouth, throat, and the nasal and oral mucosa of the upper respiratory tract [15]. Recurrent URTI takes place in subjects with chronic SIgA deficiency [16]. Also, IgA antibodies complex with antigens in the mucosal lamina propria, which finally aids in recovery from infection [17]. Such protective effect is dependent mainly on its amount and rate of secretion into the mucosa surface [18]. Thus, changes in the secretion of SIgA can be a potential indicator of the exercise-mediated effects on mucosal protection. 
SIgA is used for an objective index of the condition evaluation, including upper respiratory infections [17].

The previous study has demonstrated that 2 weeks of weight loss before a competition can impair cell-mediated immunity leading to a higher susceptibility to URTI in judo athletes [19]. The concerned and responsible authorities like coaches, support staff, assistance staff, and athletes should monitor athletes' weight loss, hydration status, the appearance of URTI symptoms, and immunocompetence such as lymphocytes and monocytes to prevent the physical condition from becoming worse [19]. Energy restriction seemed to exacerbate alterations in immune markers such as immunoglobulin and complement induced by vigorous exercise at the seventh day after a competition [20]. Similar to that, the SIgA out of saliva was found to be decreased with the appearance of upper respiratory symptoms in university rugby football players during intensive training [21]. Thus, the immune function index can be a reliable parameter to judge the wellness condition of an athlete just by simple examination of the saliva instead of blood.

The weight loss has a remarkable influence on the psychological state of the athlete characterized by exaggerated level fatigue of Profile of Mood States (POMS) and total mood disturbance (TMD) in the past study [22]. This study had shown a strong association with fatigue at various stages of competition judo athletes [1]. Other past studies indicated that the weight loss program before a competition caused a significant level of increased emotional instability, and the drop of the psychological health, including the willpower drop [7, 23]. Thus, the weight loss phase before a judo competition should be well searched from various perspectives, including physical wellbeing to mental state.

To date, a study on the influence of the condition of the weight loss using saliva SIgA has been performed [6]. However, no research has checked a change of saliva SIgA by the difference in weight loss width of the judo athlete at various time points in relation to the upper respiratory infection incidence to date before a real competition. Thus, the present study intended to examine the sequential changes of body weight, the physical and mental conditions of the athletes by the difference of weight loss width using saliva SIgA, a URTI symptom questionnaire, and POMS during the weight loss period before a real completion of judo.

\section{Materials and Methods}

\section{Subjects}

We enrolled 30 participants (22 men, eight women) who are judo players. We defined three groups as follow: 10 participants who did not undergo weight loss challenge for the competition as control group (eight men, two women); 10 participants needed under 5\% weight loss for the regulated weight as under 5\% weight loss group (seven men, three women); and 10 participants needed over 5\% weight loss for the adjusted weight as over 5\% weight loss group (seven men, three women).

\section{Ethical issues and informed consent}

All relevant information, like the purpose and methodology of the experiment, was explained to study participants beforehand, and informed consent was obtained. All procedures of the present study were conducted in compliance with the Helsinki declaration for research on human beings. The study was approved by the local research ethics committee. The study protocol was approved by the Ethical Review Board of Tsukuba University's Physical Education Faculty.

\section{Experiment procedure}

We measured the vital parameters at the third week, second week, 10th day, first week, third day, and 1 day before the competition. We did not instruct the way of weight loss. Therefore, each participant followed their usual way of weight loss method. Here as a weight-loss method, dieting, water restrictions, judo exercise, use of rubber suits during training, and sauna were used without applying any selected weight loss method. The participants were instructed and advised to not undergo intensive and exhaustive exercises for at least $24 \mathrm{~h}$ before the measurements took place. The participants fasted overnight for at least $10 \mathrm{~h}$ with suppression of caffeine consumption (allowing only caffeine-free drinks), alcohol drinking, and smoking. All measurements were conducted in a laboratory that was maintained at $24-26{ }^{\circ} \mathrm{C}$.

\section{Measurement}

\section{Body composition}

We measured height, weight, body fat percentage, and body water content. Body fat percentage and body water content were measured by multi-frequency impedance equipment (body composition meter, inner scan, 50V BC-622, Tanita). We also calculated the body mass index $\left(\mathrm{BMI}, \mathrm{kg} / \mathrm{m}^{2}\right)$ by this information.

\section{Saliva collection and analysis}

The method applied for saliva collection has already been stated in our previous report [24]. We measured collected saliva in the morning between $7 \mathrm{am}$ and $7.30 \mathrm{am}$. The study subjects have been allowed to sit, and then they washed their mouth with $30 \mathrm{~mL}$ water three times. After $5 \mathrm{~min}$ rest, the sterile sponge (Salivette, Sarstedt Inc., Rommelsdorf, Germany) was kept inside their mouth. Every subject chewed a swab of Salivette every second for $2 \mathrm{~min}$. The Salivette swabs were then centrifuged to extract saliva. The extracted saliva was weighed, and the salivary secretion rate (SSR) was determined. After the anti-secretory component antibody and the anti-IgA antibody were added to the collected saliva, enzyme-linked immunosorbent assays that specifically detected SIgA were used 
Table 1. Subject's Basic Profiles

\begin{tabular}{lllll} 
& Control group $(\mathbf{n}=\mathbf{1 0})$ & Under 5\% WL group $(\mathbf{n}=\mathbf{1 0})$ & Over 5\% WL group $(\mathbf{n}=\mathbf{1 0})$ \\
\hline Height $(\mathrm{cm})$ & $173.3 \pm 2.8$ & $168.9 \pm 3.4$ & $165.4 \pm 2.5$ & $20.1 \pm 0.3$ \\
Age $($ years $)$ & $20.3 \pm 0.4$ & $19.9 \pm 0.4$ & $68.0 \pm 3.0^{*}$ \\
Weight $(\mathrm{kg})$ & $93.7 \pm 8.8$ & $72.6 \pm 4.2$ & $24.8 \pm 0.5^{*}$ & $\mathrm{NS}$ \\
BMI $\left(\mathrm{kg} / \mathrm{m}^{2}\right)$ & $30.9 \pm 2.5$ & $25.3 \pm 0.7 *$ & $18.0 \pm 2.3$ & \\
Body fat $(\%)$ & $26.1 \pm 4.1$ & $19.9 \pm 2.7$ & $53.0 \pm 3.1$ & $\mathrm{NS}$ \\
Muscle mass $(\mathrm{kg})$ & $63.3 \pm 4.2$ & $55.0 \pm 3.9$ & $60.6 \pm 1.5^{*}$ \\
\hline Total body water $(\%)$ & $53.3 \pm 2.7$ & $57.2 \pm 1.2$ & $\mathrm{NS}$ \\
\hline
\end{tabular}

WL: weight loss; NS: not specified. ${ }^{*} \mathrm{P}<0.05$ versus control group.

to determine the SIgA concentrations (SIgA-Cs). The SIgA secretion velocity (SIgA-V) was calculated as follows:

$$
\begin{gathered}
\text { SIgA-V }(\mu \mathrm{g} / 2 \mathrm{~min})=\text { Salivary flow rate } \\
(\mathrm{mL} / 2 \mathrm{~min}) \times \operatorname{SIgA}-\mathrm{C}(\mu \mathrm{g} / \mathrm{mL})
\end{gathered}
$$

\section{Saliva flow rate}

The quantity of saliva measured weight (g) of saliva secreted for $2 \mathrm{~min}$, and the specific gravity of saliva calculated amount of saliva per $2 \mathrm{~min}$ as $1.0 \mathrm{~g} / \mathrm{mL}(\mathrm{mL} / 2 \mathrm{~min})$.

\section{The screening test of URTI symptoms}

To screen the URTI, we followed the previous article [25] and made a questionnaire. The questionnaire contained six items, namely on headache, sore throat, cough, mucus, running nose, and lassitude. We asked the participants to check their physical condition according to the questionnaire and determined the prevalence of the above symptoms 3 weeks to 1 day before the competition.

\section{POMS}

We used the Japanese version of POMS that contains Tension or Anxiety, Anger or Hostility, Vigor or Activity, Fatigue or Inertia, Depression or Dejection and Confusion, or Bewilderment. We calculated the TMD score by each score and analyzed each cohort [26].

\section{Statistics}

The results were expressed as mean \pm standard error (SE). Two-way analysis of variance (two-way ANOVA) was used for the analysis, and the Bonferroni method was used for post hoc analysis. The study subjects' basic profiles in Table 1 were analyzed by one-way ANOVA. All statistical analyses were performed using SPSS version 25 for Mac OS X (SPSS Inc., Chicago, IL, USA), and $\mathrm{P}<0.05$ was considered statistically significant for all parameters.

\section{Results}

\section{Changes in body weight before competition}

Table 1 showed the essential biological characteristics of study participants. We showed the mean body weight value among the study groups in Table 1. For body weight, the mean weight was statistically significant in the group losing more than $5 \%$ body weight in comparison to that of control group. There were also significant changes observed in context to BMI (Table 1) in both the body weight loss groups, either less than $5 \%$ or more than $5 \%$ in comparison to the control group.

Figure 1 demonstrated the time course of body weight changes in study participants. The interaction between control group, under 5\% weight loss group and over 5\% weight loss group was significantly different $(\mathrm{F}(10,135)=11.8, \mathrm{P}$ $\left.<0.001, \eta^{2}=0.466\right)$. For the group losing less than $5 \%$ body weight before completion, only 1 day before the competition, the body weight was significantly low compared to that of the control group $(\mathrm{P}<0.05)$. Within the under $5 \%$ body weight loss group, at day 3 and day 1 before the competition the body was significantly changed in comparison to that of 3 weeks before the competition, and at the day before the competition there was a significant change in body weight compared with 2 weeks before the competition $(\mathrm{P}<0.05)$. There was no intragroup difference in body weight among the various time points measured in the current study in the control group. In

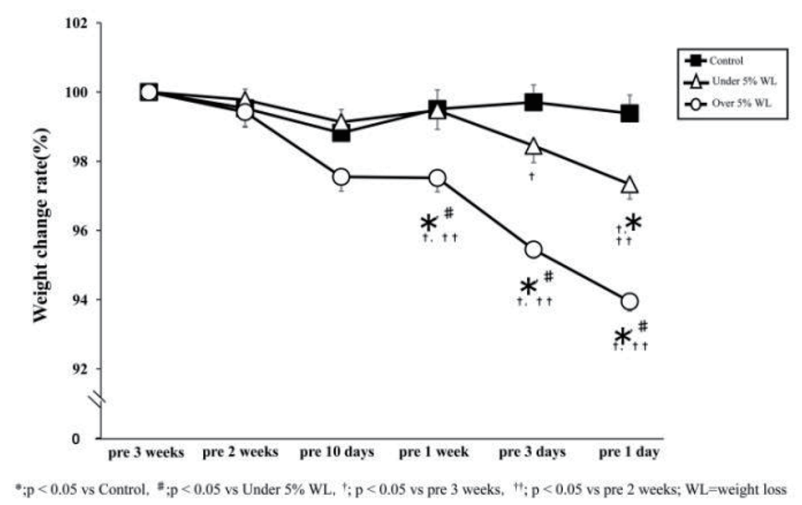

Figure 1. Weight change rate. WL: weight loss. 


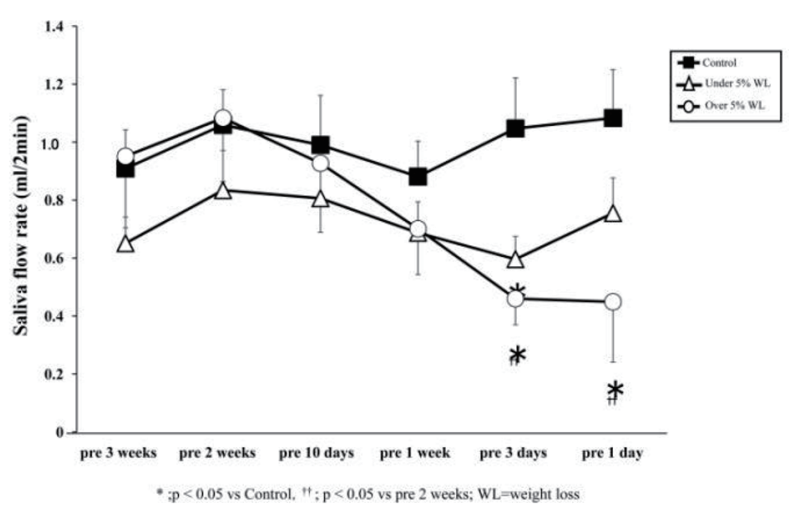

Figure 2. Saliva flow rate. WL: weight loss.

comparison to the control group and under 5\% body weight loss group, body weight was significantly reduced in the group losing more than $5 \%$ body weight at time points of 1 week, 3 days and 1 day before the competition. Within the same group of more than $5 \%$ body weight loss, in comparison to the time points of 2 weeks and 3 weeks before a competition, the closest time points before competition namely the 1 week, 3 days and the day before showed significant reduction of body weight loss $(\mathrm{P}<0.05)$.

\section{Changes in salivary parameters}

We also showed the change of the saliva flow rate, which means volume of the saliva before competition at various time points in Figure 2. The interaction among the three groups (control, under $5 \%$ body weight loss and over $5 \%$ body weight loss) of total volume of saliva was $\left(\mathrm{F}(10,135)=2.8, \mathrm{P}<0.004, \eta^{2}=\right.$ $0.171)$. In comparison between groups, the total volume of the saliva of under $5 \%$ group showed a significant decrease at the third day before the competition than that of control group ( $\mathrm{P}$ $<0.05)$. When under $5 \%$ group showed a significant reduction in saliva flow rate only at a one-time point, over 5\% group showed a considerable drop at two-time points before competition including 1 day and 3 days before compared to control

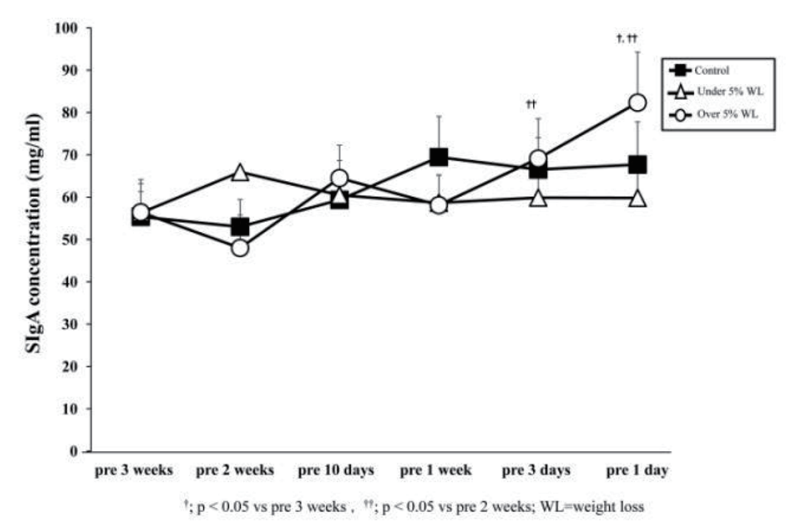

Figure 3. SIgA concentration. WL: weight loss; SIgA: secretory immunoglobulin A.

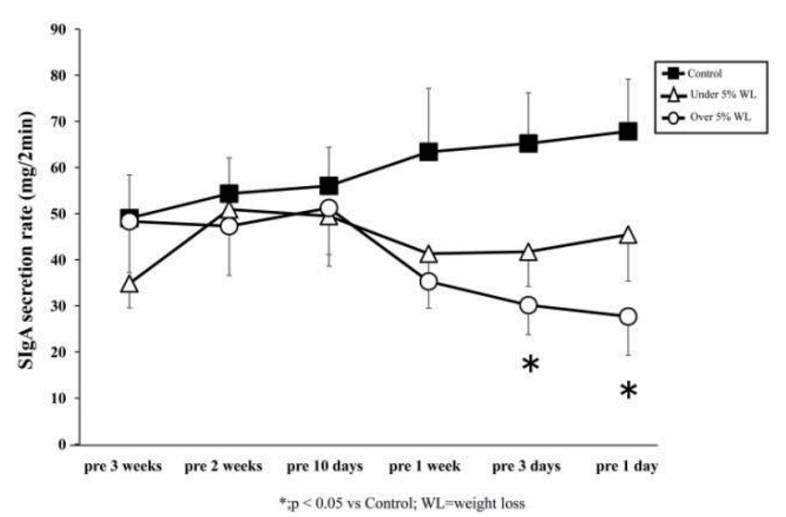

Figure 4. SIgA secretion rate. WL: weight loss; SIgA: secretory immunoglobulin A.

group $(\mathrm{P}<0.05)$. Within the over 5\% body weight loss group, the total volume of saliva significantly decreased at day 3 and day 1 before the competition when compared with the 2 weeks before the competition in the same group $(\mathrm{P}<0.05)$. These intragroup differences were not observed within the under $5 \%$ body weight loss group and the control group (Fig. 2). Figure 3 shows the SIgA-C among the three study groups at various time points examined. The interaction among study groups of the $\operatorname{SIgA-C}$ was $\left(\mathrm{F}(10,135)=2.5, \mathrm{P}<0.008, \eta^{2}=0.158\right)$. Within the over 5\% body weight loss group, the SIgA-C significantly increased at day 3 before the competition when compared with 2 weeks before the competition $(\mathrm{P}<0.05)$, and also the day before the competition compared with 2 weeks and 3 weeks before the competition $(\mathrm{P}<0.05)$. These significant differences were not observed within the under $5 \%$ body weight loss group and the control group.

Figure 4 shows the SIgA-V in saliva at various time points among study groups. At the third day and the day before the competition, compared to the control group there was a significant reduction in $\mathrm{SIgA}-\mathrm{V}$ in over $5 \%$ body weight loss group $(\mathrm{P}<0.05)$. When the intragroup comparison was made for each respective group at various time points, there was no substantial change observed. Additionally, in between-group comparisons of body weight loss more than or less than $5 \%$, no considerable difference was seen at various time points.

\section{Incidence of URTI symptoms among study participants}

We showed the incidence of the URTI symptoms among study subjects at various time points before the real competition held (Fig. 5). The incidence of the URTI symptoms at the day before the competition in under $5 \%$ body weight loss group was as follows: one case of headache; one case of sore throat; two cases of lassitude. The incidence of the URTI symptoms at the day before the competition in over $5 \%$ body weight loss group was: one case of headache; three cases of sore throat; five cases of the running nose; six cases of lassitude. There was no incidence of the URTI symptoms in the control group. 


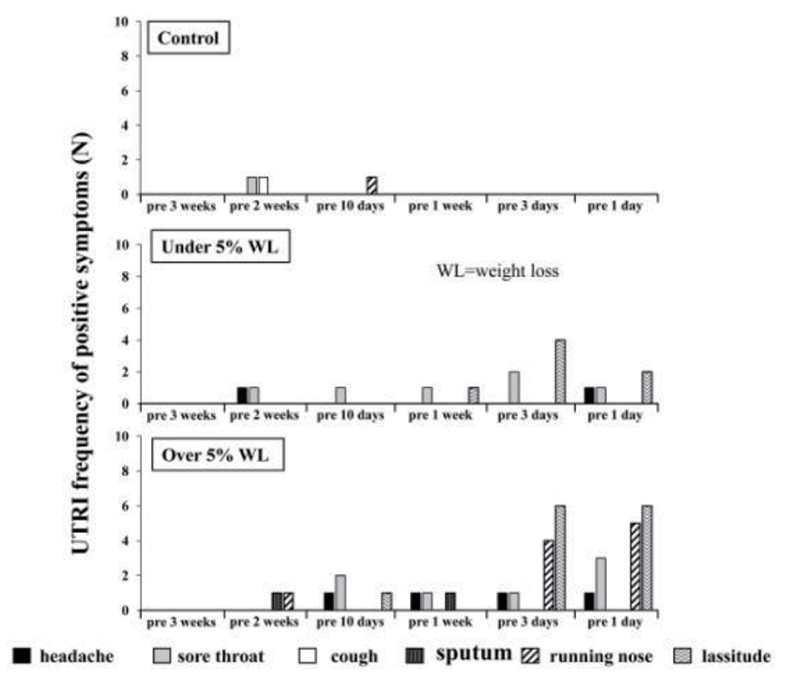

Figure 5. URTI frequency of positive symptoms. URTI: upper respiratory tract infection.

\section{The psychological condition of study subjects}

We showed the result of POMS before the competition at various time points among study groups in Table 2 . The interaction among groups (control, under $5 \%$ body weight loss group and over $5 \%$ body weight loss group) for vigor ( $\mathrm{F}(6$, $\left.81)=5.8, \mathrm{P}<0.001, \eta^{2}=0.296\right)$ and that of fatigue $(\mathrm{F}(6$, $81)=2.9, P<0.04, \eta^{2}=0.134$ ) were significantly different at statistical level. In case of vigor, there was a significant intragroup difference in the group of more than $5 \%$ body weight loss characterized by a considerable decrease of vigor at the day before the competition compared with those of 3 weeks and 2 weeks before the competition $(\mathrm{P}<0.05)$. Similarly, the fatigue level in more than $5 \%$ body weight loss group was significantly increased before the day of the competition compared with 3 and 2 weeks before the competition $(\mathrm{P}<0.05)$. There was no significant difference in context to tension, depression, confusion, and TMD at all the observed periods of study groups.

\section{Discussion}

The current study investigated the gradual changes in body weight, parameters of saliva including volume, the concentration and secretion rate of the SIgA, the incidence of URTI symptoms, and psychological condition levels at various time points during weight loss period before a real competition among university judo athletes with a focus on body weight decline range. This is the first study showing the immunological status demonstrated through SIgA in correlation to the incidence rate of URTI symptoms among judo athletes in comparison to more or less than 5\% body weight reduction before the real competition with a time profiling. A significant decrease in SIgA secretion rate and saliva flow speed was observed in the group having a body weight loss of more than $5 \%$ compared to the control group. These changes in saliva were accompanied by the si- multaneous association of incidence of the URTI symptoms and impaired psychological condition. Therefore, the over 5\% weight loss of the judo athlete before competition caused a drop of oral immunity and the disturbed mental state, which are not beneficial things for judo athlete before a competition.

In the current study, there was no artificial body weight loss program followed. All participants lost their body weight in their habitual natural usual ways before the competition. Also, there was no drastic or rapid weight loss protocol applied. As evident in Figure 1, there was a gradual weight loss to achieve the best fitness for the competition. Therefore, the result of this study possesses methodological validity because there is no external bias, and we assume that this study well describes the natural change of the immune system before competition for a judo player with a weight-loss period.

Assessment of salivary immunoglobulins, especially SIgA and other associated antimicrobial proteins, successfully represents the effects of various exercises on mucosal immunity [27]. Increases in SIgA and antimicrobial protein concentration and secretion rate have a significant association with acute exercise, whereas, in contrast, decreases have been reported in athletes over a training season, leading the athlete susceptible to URTI. The measurement of physiological biomarkers in the whole saliva can provide an essential tool for measuring the immunological and endocrinological status associated with exercise and training [28]. Indeed, the more specifically past study had identified the time course of change of salivary testosterone, cortisol, and IgA along with changes in mood state; and performance capacity during a 2-week taper in judo athletes and a diurnal variation in these salivary markers [29] were observed. Other studies demonstrated that continuous highly intensive training reduces SIgA secretion speed at rest $[25,27,30-32]$. Also, it is clearly shown that exercise-induced weight loss induced a significant decrease in the SIgA flow rate $[6,33,34]$. Thus, a differential pattern on SIgA was observed in different studies in a different setting.

Here, we observed the decreased volume of saliva during the weight loss phase with a significant drop only 3 days before and the day before the competition in more than $5 \%$ body weight loss group in comparison to the control group. Also, in the same weight loss group, there was a significant drop in saliva flow rate 3 days before and 1 day before the competition compared to that observed 2 weeks before the game. This phenomenon might reflect the dehydration status during the weight-loss period approaching competition due to a weight loss of more than $5 \%$. We also observed an increase in the concentration of SIgA in more than $5 \%$ body weight loss group 3 days and 1 day before the competition, which was accompanied by a simultaneous decrease in secretion capacity of SIgA in saliva. It seems a paradoxical change but can be explained when considering dehydration as a confounder for this phenomenon. Previous studies showed the association between weight loss by training and an increase in the concentration of SIgA in saliva due to dehydration [35]. This study is in line with the findings of the current research having similar types of association between weight loss and the concentration of SIgA. Fortes et al demonstrated the complementary outcome that participants who exercised 
Table 2. POMS's Fluctuation

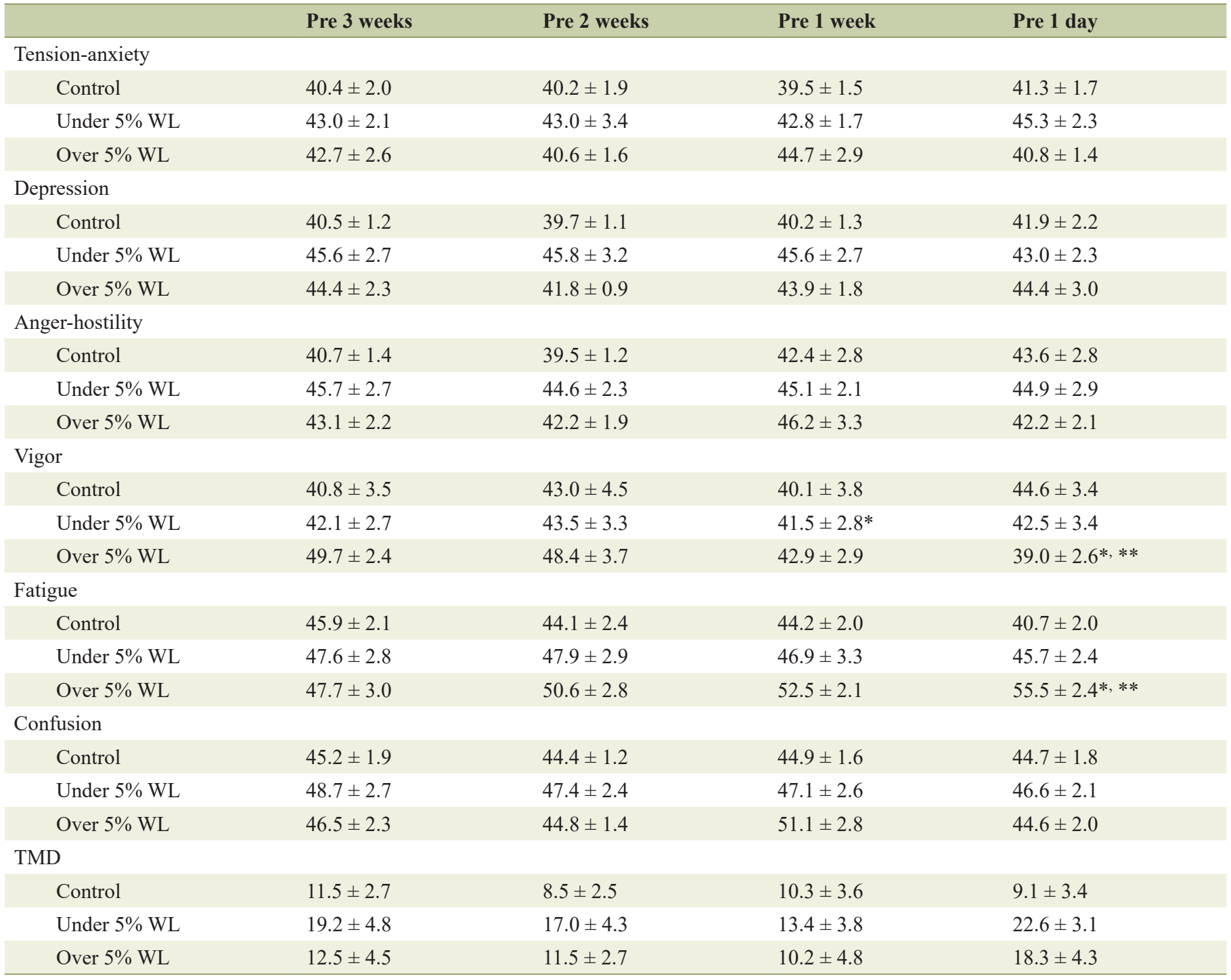

WL: weight loss; TMD: total mood disturbance; POMS: Profile of Mood States. ${ }^{*} \mathrm{P}<0.05$ versus pre 3 weeks; ${ }^{*} \mathrm{P}<0.05$ versus pre 2 weeks.

in the heat, without fluid intake to evoke progressive body mass losses of $3 \%$ with subsequent overnight fluid restriction, had increased urinary specific gravity and SIgA concentration [33] implying a potential effect of dehydration as an underlying mechanism.

A decrease in the saliva concentration of SIgA has been implicated as a possible causal and contributing factor in the increased susceptibility to URTI during heavy training in athletes [36, 37]; however, the present study does not support those results. In line with our current finding, some studies have observed an acute increase in SIgA in response to exercise [27], and there is speculation that this increase might be attributed to $\beta$-sympathetic actions on the salivary glands [38]; therefore, the increase in the present study could perhaps represent an increased sympathetic nervous system activation in response to exercise and weight loss. In an attempt to explain the findings, there might be the fact that with an increased saliva flow rate one can expect a decrease in $\mathrm{SIgA}$ secretion due to a dilution effect [27], but there was an increase in the secretion during this period, which would be due to a rise in absolute concentration. An alternative explanation could be in linkage to the psychological strain on the athletes as a result of the competition. From the present findings, SIgA concentration might show an increase by having performed rapid weight loss including the occurrence of the dehydration at the time points approaching to the day of competition accompanied with intensive shortterm weight loss width in the over $5 \%$ weight loss group where the quantity of saliva showed a drop before the game.

In the current study, the incidence of URTI symptoms increases, and psychological conditions, including fatigue and loss of vigor, are deteriorated in more than $5 \%$ weight loss group at the day before the competition. Increased incidence of URTI symptoms may reflect and represent the immune suppression with body weight loss. Again, the suppression state of immu- 
nity may potentially impact the fatigue level and loss of vigor. Several studies have revealed the active association between the psychological condition and immune systems [39-41]. Notably, a recent study showed a relationship among the alteration of immunity, inflammation in central nervous systems, and psychiatric disorders [42]. There was a vice-versa relation observed between major depressive disorder (MDD) and inflammation [43]. There are a general conception and image that elite athletes have a healthy mind, but a recent study provided a vulnerability picture of mental health disturbance among these sports athletes $[44,45]$. World Health Organization reported that MDD affected over 300 million peoples all over the world in 2018 [46] and Doherty et al reported MDD during the careers of elite male athletes by a qualitative study [47].

There are several limitations to the current study. Firstly, the study was confined to judo players from only one university; therefore, it is hard to generalize the effects to other judo players. We did not assess the dehydration status directly in the current study design. Circulatory, immune profile measurement can complement the strength of current research findings. Using a study design that compares simultaneously the immune profile with both the natural weight loss protocol and the rapid weight loss manual can comprehend the mechanistic insight underlying the current study. Further research is needed to explore the direct association of performance and immune suppression caused by weight loss during the competition, including both physical and psychological conditions with a long-range follow-up study.

\section{Conclusions}

The current findings for the first time demonstrate that saliva SIgA and the upper respiratory infection symptoms are differentially affected by the difference of the weight loss width before the competition of the university judo athletes. In comparison to the control group, there is a dropping tendency of SIgA and a risk of the upper respiratory infection incidence in over 5\% weight loss group with the deterioration of the psychological condition. Therefore, the weight loss program range should be made and tailored with great caution and care for judo athletes for better performance during competition.

\section{Acknowledgments}

We appreciate the support of the Kozuki Foundation for Sports.

\section{Financial Disclosure}

There was no specific funding source to be mentioned.

\section{Conflict of Interest}

The authors declare that they have no conflict of interest.

\section{Informed Consent}

All subjects provided written informed consent.

\section{Author Contributions}

$\mathrm{HH}$ has designed and performed the study. $\mathrm{HH}, \mathrm{SJ}$, and $\mathrm{KW}$ have drafted the manuscript and did critical editing. YH, FK, $\mathrm{YM}$, and KS have assisted and supported in sample collection and subsequent analysis with statistics. SJ and KW have carefully supervised this manuscript preparation and writing.

\section{References}

1. Hiraoka H, Ishi T, Hanaoka Y, Ono T, Akimoto H, Kobayasi Y, Suzuki N, et al. Effects of weight loss on the psychological condition of male national and university judoists before the competition. Sport Science Research. 2018;15:88-99.

2. Filaire E, Maso F, Degoutte F, Jouanel P, Lac G. Food restriction, performance, psychological state and lipid values in judo athletes. Int J Sports Med. 2001;22(6):454-459.

3. Kurakake S, Umeda T, Nakaji S, Sugawara K, Saito K, Yamamoto Y. Changes in physical characteristics, hematological parameters and nutrients and food intake during weight reduction in judoists. Environ Health Prev Med. 1998;3(3):152-157.

4. Franchini E, Del Vecchio FB, Matsushigue KA, Artioli GG. Physiological profiles of elite judo athletes. Sports Med. 2011;41(2):147-166.

5. Hanaoka Y, Shimizu K, Washiya K, Tamura A, Takemura M, Furukawa T, Miyamoto T, et al. The effects of Lactobacillus gasseri OLL2809 intake on oral immune function in college rugby athletes. The Japanese Journal of Physical Fitness and Sports Medicine. 2015;64(3):315-322.

6. Shimizu K, Aizawa K, Suzuki N, Kukidome T, Kimura F, Akama T, Mesaki N, et al. Evaluation of condition during rapid weight loss using salivary level of secretory SIgA in elite wrestlers. The Journal of Clinical Sports Medicine. 2007;15(3):441-447.

7. Tsuya R, Umeda T, Mochida N, Kojima A, Yamamoto Y, Manabu T, Yoshioka Y, et al. The relationship between changes in anthropometric parameters and nutritional intake and mental stress in college judoists during weight reduction periods. -Differences between male and female judoists. Journal of Physical Fitness, Nutrition and Immunology. 2003;13:31-39.

8. Umeda T, Nakaji S, Sugawara K, Yamamoto Y, Saito K, Honjo S, Sakurai Y, et al. Gender differences in physical and psychological stress responses among college judoists undergoing weight reduction. Environ Health Prev Med. 1999;4(3):146-150.

9. Artioli GG, Gualano B, Franchini E, Scagliusi FB, Takesian M, Fuchs M, Lancha AH, Jr. Prevalence, magnitude, and methods of rapid weight loss among judo competitors. Med Sci Sports Exerc. 2010;42(3):436-442. 
10. Alderman B, Landers DM, Carlson J, Scott JR. Factors related to rapid weight loss practices among international-style wrestlers. Med Sci Sports Exerc. 2004;36(2):249252.

11. Kiningham RB, Gorenflo DW. Weight loss methods of high school wrestlers. Med Sci Sports Exerc. 2001;33(5):810-813.

12. Oppliger RA, Steen SA, Scott JR. Weight loss practices of college wrestlers. Int J Sport Nutr Exerc Metab. 2003;13(1):29-46.

13. Steen SN, Brownell KD. Patterns of weight loss and regain in wrestlers: has the tradition changed? Med Sci Sports Exerc. 1990;22(6):762-768.

14. Walsh NP, Gleeson M, Shephard RJ, Gleeson M, Woods JA, Bishop NC, Fleshner M, et al. Position statement. Part one: Immune function and exercise. Exerc Immunol Rev. 2011;17:6-63.

15. Mackinnon LT, Hooper SL. Plasma glutamine and upper respiratory tract infection during intensified training in swimmers. Med Sci Sports Exerc. 1996;28(3):285-290.

16. Reid MR, Mackinnon LT, Drummond PD. The effects of stress management on symptoms of upper respiratory tract infection, secretory immunoglobulin A, and mood in young adults. J Psychosom Res. 2001;51(6):721-728.

17. Lamm ME, Nedrud JG, Kaetzel CS, Mazanec MB. IgA and mucosal defense. APMIS. 1995;103(4):241-246.

18. Mackinnon LT, Hooper S. Mucosal (secretory) immune system responses to exercise of varying intensity and during overtraining. Int J Sports Med. 1994;15(Suppl 3):S179-183.

19. Shimizu K, Aizawa K, Suzuki N, Masuchi K, Okada H, Akimoto T, Mesaki N, et al. Influences of weight loss on monocytes and T-cell subpopulations in male judo athletes. J Strength Cond Res. 2011;25(7):1943-1950.

20. Umeda T, Nakaji S, Shimoyama T, Kojima A, Yamamoto Y, Sugawara K. Adverse effects of energy restriction on changes in immunoglobulins and complements during weight reduction in judoists. J Sports Med Phys Fitness. 2004;44(3):328-334.

21. Yamauchi R, Shimizu K, Kimura F, Takemura M, Suzuki K, Akama T, Kono I, et al. Virus activation and immune function during intense training in rugby football players. Int J Sports Med. 2011;32(5):393-398.

22. Helms ER, Zinn C, Rowlands DS, Naidoo R, Cronin J. High-protein, low-fat, short-term diet results in less stress and fatigue than moderate-protein moderate-fat diet during weight loss in male weightlifters: a pilot study. Int J Sport Nutr Exerc Metab. 2015;25(2):163-170.

23. Horswill CA, Hickner RC, Scott JR, Costill DL, Gould D. Weight loss, dietary carbohydrate modifications, and high intensity, physical performance. Med Sci Sports Exerc. 1990;22(4):470-476.

24. Hiraoka H, Hanaoka Y, Shimizu K, Okada H, Masuchi $\mathrm{K}$, Watanabe K. Effects of immune function with weight loss in judo athletes during training camp. The Journal of Clinical Sports Medicine. 2018;26(1):100-108.

25. Neville V, Gleeson M, Folland JP. Salivary IgA as a risk factor for upper respiratory infections in elite professional athletes. Med Sci Sports Exerc. 2008;40(7):1228-1236.
26. McNair DM, Lorr M, Droppelman LF. Profile of Mood States Manual. San Deigo, Calif: Educational and Industrial Testing Service. 1992.

27. Otsuki T, Shimizu K, Iemitsu M, Kono I. Chlorella intake attenuates reduced salivary SIgA secretion in kendo training camp participants. Nutr J. 2012;11:103.

28. Papacosta E, Nassis GP. Saliva as a tool for monitoring steroid, peptide and immune markers in sport and exercise science. J Sci Med Sport. 2011;14(5):424-434.

29. Papacosta E, Gleeson M, Nassis GP. Salivary hormones, IgA, and performance during intense training and tapering in judo athletes. J Strength Cond Res. 2013;27(9):25692580 .

30. Akimoto T, Akama T, Kodo Y, Waku T, Hayashi E, Tatsuno M, Sugiura K, et al. Effects of repetitious intense exercise training on resting salivary IgA. The Japanese Journal of Physical Fitness and Sports Medicine. 1998;47:245-251.

31. Tharp GD, Barnes MW. Reduction of saliva immunoglobulin levels by swim training. Eur J Appl Physiol Occup Physiol. 1990;60(1):61-64.

32. Ymauchi R, Shimizu K, Furukawa T, Watanabe K, Takemura M, Akama T, Akimoto T, et al. Alterations of salivary SIgA during training camp in collegiate rugby football players. The Japanese Journal of Physical Fitness and Sports Medicine. 2009;58(1):131-142.

33. Fortes MB, Diment BC, Di Felice U, Walsh NP. Dehydration decreases saliva antimicrobial proteins important for mucosal immunity. Appl Physiol Nutr Metab. 2012;37(5):850-859.

34. Tsai ML, Ko MH, Chang CK, Chou KM, Fang SH. Impact of intense training and rapid weight changes on salivary parameters in elite female Taekwondo athletes. Scand J Med Sci Sports. 2011;21(6):758-764.

35. Oliver SJ, Laing SJ, Wilson S, Bilzon JL, Walsh NP. Saliva indices track hypohydration during $48 \mathrm{~h}$ of fluid restriction or combined fluid and energy restriction. Arch Oral Biol. 2008;53(10):975-980.

36. Gleeson M, McDonald WA, Pyne DB, Cripps AW, Francis JL, Fricker PA, Clancy RL. Salivary IgA levels and infection risk in elite swimmers. Med Sci Sports Exerc. 1999;31(1):67-73.

37. Gleeson M, McDonald WA, Cripps AW, Pyne DB, Clancy RL, Fricker PA. The effect on immunity of long-term intensive training in elite swimmers. Clin Exp Immunol. 1995;102(1):210-216.

38. Chicharro JL, Lucia A, Perez M, Vaquero AF, Urena R. Saliva composition and exercise. Sports Med. 1998;26(1):17-27.

39. Schaller M. The behavioural immune system and the psychology of human sociality. Philos Trans R Soc Lond B Biol Sci. 2011;366(1583):3418-3426.

40. D'Acquisto F. Affective immunology: where emotions and the immune response converge. Dialogues Clin Neurosci. 2017;19(1):9-19.

41. Abdurachman A, Herawati N. The role of psychological well-being in boosting immune response: an optimal effort for tackling infection. Afr J Infect Dis. 2018;12(S):5461 . 
42. Mondelli V, Dazzan P, Pariante CM. Immune abnormalities across psychiatric disorders: clinical relevance. BJPsych Adv. 2015;21(3):150-156.

43. Amodeo G, Allegra Trusso M, Fagiolini A. Depression and inflammation: disentangling a clear yet complex and multifaceted link. Neuropsychiatry (London). 2018;07(04):448-457.

44. Sebbens J, Hassmen P, Crisp D, Wensley K. Mental Health in Sport (MHS): improving the early intervention knowledge and confidence of elite sport staff. Front Psychol. 2016;7:911.

45. Rice SM, Purcell R, De Silva S, Mawren D, McGorry PD, Parker AG. The mental health of elite athletes: a narrative systematic review. Sports Med. 2016;46(9):1333-1353.

46. Organization WHO. Depression. 2018.

47. Doherty S, Hannigan B, Campbell MJ. The Experience of depression during the careers of elite male athletes. Front Psychol. 2016;7:1069. 